Gynäkologe 2016 · 49:904-905

DOI 10.1007/s00129-016-3990-2

Online publiziert: 11 . November 2016

(c) Springer Medizin Verlag Berlin 2016

CrossMark

\author{
N. Maass ${ }^{1} \cdot$ W. Janni ${ }^{2}$ \\ 'Klinik für Gynäkologie und Geburtshilfe, Kiel, Deutschland \\ ${ }^{2}$ Universitätsklinikum Ulm, Ulm, Deutschland
}

\title{
Operative Therapien in der Gynäkologie
}

\section{Segen und Verpflichtung von Innovationen}

Wir schreiben das Jahr 2007. Nokia ist der größte Mobilfunkkonzern der Welt und das finnische Betriebssystem Symbian hat einen Marktanteil von deutlich über $60 \%$. Das ist zwar keine Monopolstellung, aber man kann an dieser Stelle schon von einer marktdominierenden Dominanz sprechen. Die Handywelt war also fest in finnischer Hand und die Produkte von Nokia waren beliebt und so etwas wie „State of the Art“. Ab diesem Zeitpunkt war es dramatisch zu erleben, wie die Marktanteile dieses Herstellers sich quartalsweise immer weiter reduzierten bis zum Nullpunkt. Die Erfolgsgeschichte von Smartphones, die vermutlich alle Leser dieses Heftes heute so natürlich begleiten wie die Zahnbürste auf Reisen, ist uns sicher allen bekannt. Ein Leben ohne die Innovation des Smartphones ist heute nur noch schwer vorstellbar und baut sich uns erst bei schmerzhaftem Verlust des entsprechenden Gerätes als Drohkulisse auf.

Dieses nichtmedizinische Phänomen macht uns bewusst, wie sehr Innovationen unser Leben und unseren Alltag beeinflussen können, bis hin zu Normalitäten und Selbstverständlichkeiten. Gleichzeitig macht es uns aber auch bewusst, wie hart in einer sich dynamisch entwickelnden Welt jene bestraft werden, die sich nicht aufmerksam, zeitnah und erfolgreich genug innovativen Veränderungen stellen. Gleichzeitig mag dieses Beispiel aber auch deutlich machen, dass Innovationen, die zu schnell und zu unkritisch vorangetrieben werden, buchstäblich in Flammen aufgehen können. Wie ein anderer Mobiltelefonhersteller vor nicht allzu langer Zeit schmerzlich erfahren musste.

Das Fachgebiet der operativen Gynäkologie ist gesegnet mit zahlreichen Innovationen der letzten Jahrzehnte. Unser Fachgebiet ist de facto die Wiege der minimalinvasiven Chirurgie, sowohl durch die vaginalen Zugangswege, die wohl früheste Form eines NOTES-Zuganges und durch die Pionierarbeit von Professor Semm in Kiel im Bereich der Endoskopie. Seither gibt es zahlreiche Entwicklungen in der operativen Gynäkologie zu berichten, die von uns gestaltet wurden und weiter gestaltet werden müssen. Es mag damit ein guter Zeitpunkt für eine kritische Reflexion zum aktuellen Stand und zur weiteren Entwicklung der operativen Gynäkologie mit Blick auf Innovationen sein.

Die Kollegen Alkatout und Maas werfen einen Blick auf die wohl neuste, faszinierendste, aber auch streitbarste Entwicklung in der operativen Gynäkologie, die roboterassistierte Chirurgie. Hier für unser Fach das richtige Maß an Expertise, aber auch kritischer Standortbestimmung zu finden, wird auch für die nächsten Jahre eine Herausforderung sein. Die Kollegen Pecks und Schem diskutieren die beiden historisch sequenziell entstandenen Zugangswege für minimalinvasive Chirurgie in der Urogynäkologie, das vaginale und laparoskopische Vorgehen, mit ihren Vor- und Nachteilen. Die Kollegen de Gregorio und Ebner werfen einen abwägenden Blick auf eine Operationsweise, die man schon fast im Antiquariat der Operationsatlanten glaubte und doch einen weiterhin aktuellen Stellenwert hat. Die Kollegen Liedtke und Widschwendter 


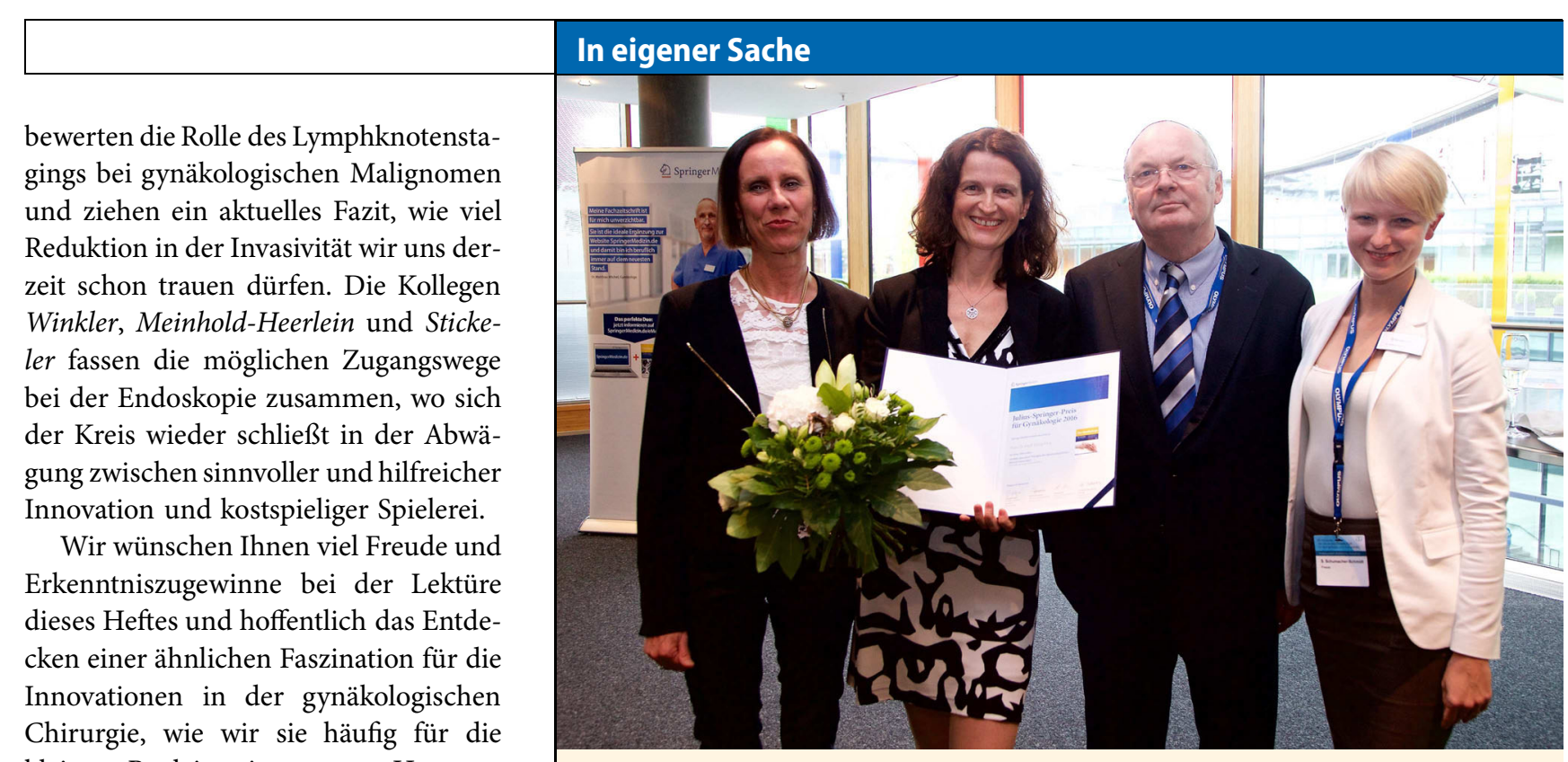
kleinen Begleiter in unserer Hosentasche empfinden. Die Innovationskraft unseres Faches weiter aufrechtzuerhalten, ist nicht nur unsere Pflicht bei der Optimierung der Krankenversorgung, sondern auch die beste Medizin für eine prosperierende Zukunft unseres Faches.

Mit herzlichen Grüßen aus Kiel und Ulm,

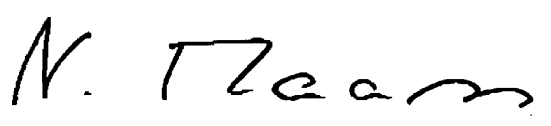

N. Maass

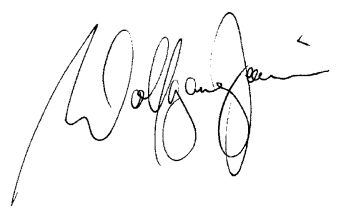

W. Janni

\section{Korrespondenzadresse}

Univ.-Prof. Dr. W. Janni
Universitätsklinikum Ulm
Prittwitzstr. 43, 89075 Ulm,
Deutschland
wolfgang.janni@uniklinik-
ulm.de

Interessenkonflikt. N. Maass und W. Janni geben an, dass kein Interessenkonflikt besteht.

v.I.n.r.: M. Kretz, V. Fink, K. Diedrich, S. Schumacher-Schmidt

\section{Julius-Springer-Preis für Gynäkologie}

Erstmals verliehen

Der beste CME-Artikel aus den beiden Springer-Fachzeitschriften Der Gynäkologe und Gynäkologische Endokrinologie wurde erstmalig ausgezeichnet. Die Preisarbeit steht für exzellenten Transfer wissenschaftlicher Ergebnisse in die medizinische Praxis.

Springer Medizin vergab 2016 erstmals einen Preis für den besten CME-Artikel in der ärztlichen Fortbildung auf dem Fachgebiet der Gynäkologie. Aus den Jahrgängen 2014 und 2015 der Zeitschriften Der Gynäkologe und Gynäkologische Endokrinologie wurde die Arbeit „Update operative Therapie des Mammakarzinoms - Wie viel muss sein?", erschienen in Der Gynäkologe 09/2014 (Rubrik CME), mit dem JuliusSpringer-Preis für Gynäkologie ausgezeichnet. Der Preis ist mit Euro 2.500,- dotiert. Am Nachmittag des 20. Oktobers nahm die Preisträgerin Dr. Visnja Fink die Auszeichnung auf dem Kongress der Deutschen Gesellschaft für Gynäkologie und Geburtshilfe (DGGG) in Stuttgart entgegen.

„Erfreulicherweise befand die Jury bei der Entscheidung über den ersten auszulobenden CME-Beitrag, dass ihr ausnahmslos Arbeiten in sehr hoher Qualität vorlagen“, betonte der Reproduktionsmediziner und Senior Editor von Der Gynäkologe, Professor Klaus Diedrich. „Dies hatte allerdings zur Folge, dass die Ergebnisse der Jury-Auswertung in den Kategorien Aktualität, Fortbildungswert, Didaktik und Darstellung recht nah beieinander lagen. Dennoch war der CMEBeitrag von Visnja Fink in allen Punkten füh- rend und überzeugte damit die Jury." Den ersten Julius-Springer-Preis für Gynäkologie erhielt die Fachärztin für ihre aktuelle Übersicht über die operativen Therapien bei Brustkrebs. Die Gynäkologin berücksichtigt hierbei sowohl brusterhaltende Verfahren als auch Operationstechniken zur Entfernung der Brust.

Dr. med. Visnja Fink ist Humanmedizinerin und arbeitete nach ihrer Promotion in verschiedenen Positionen an der Frauenklinik der LMU München. Im Oktober 2006 absolvierte sie erfolgreich die Prüfung zur Fachärztin für Gynäkologie und sammelte praktische Erfahrungen in der plastischen Brustsprechstunde und in der diagnostischen Mammasprechstunde. Seit Mai 2013 leitet die Expertin die Brustchirurgie der Universitätsfrauenklinik UIm, seit 2015 hat Fink zudem die dortige Stellvertretung des Brustzentrums inne.

\section{Foto: $\odot$ KelCon GmbH (mit freundlicher} Genehmigung, all rights reserved) 